\title{
La Locride de l'Ouest et la première forme urbaine de la ville de Naupacte
}

West Locris and the City of Nafpaktos in Iron Age

\section{Despina Chatzivasiliou}

\section{(2) OpenEdition}

1 Journals

Édition électronique

URL : https://journals.openedition.org/gaia/1188

DOI : 10.4000/gaia. 1188

ISSN : 2275-4776

Éditeur

UGA Éditions/Université Grenoble Alpes

\section{Édition imprimée}

ISBN : 978-2-37747-199-7

ISSN : 1287-3349

\section{Référence électronique}

Despina Chatzivasiliou, "La Locride de l'Ouest et la première forme urbaine de la ville de Naupacte », Gaia [En ligne], 22-23 | 2020, mis en ligne le 30 juin 2020, consulté le 09 décembre 2021. URL : http:// journals.openedition.org/gaia/1188; DOI : https://doi.org/10.4000/gaia.1188

Ce document a été généré automatiquement le 9 décembre 2021.

Gaia. Revue interdisciplinaire sur la Grèce archaïque 


\title{
La Locride de l'Ouest et la première forme urbaine de la ville de Naupacte
}

West Locris and the City of Nafpaktos in Iron Age

\author{
Despina Chatzivasiliou
}

Dans le foisonnement des études consacrées aux formes d'urbanisation de l'âge du fer en Grèce, la Locride de l'Ouest a peu attiré l'attention des chercheurs ${ }^{1}$. Le choix d'étudier les étapes de l'organisation urbaine dans une région peu connue s'avère d'autant plus intéressant que la bibliographie existante sur le sujet permet de lancer un regard neuf. Par la présente étude, nous ne prétendons pas épuiser le sujet dans tous ses aspects, mais proposer une lecture avec des idées qui dérivent de la description géographique, des sources littéraires et des vestiges archéologiques. Cette approche s'intéresse surtout à la définition de l'espace, à la formation des territoires et à la structuration des centres urbains. Le raisonnement se développe en trois parties: d'abord définir à quelle zone géographique correspond la Locride, ensuite décrire la Locride de l'Ouest et enfin présenter plus précisément nos données sur la ville de Naupacte qui nous sert de cas d'étude.

\section{La Locride ou les Locrides}


Fig. 1. - Carte de la Grèce centrale avec les régions de la Locride de l'Est et de l'Ouest et les régions limitrophes.

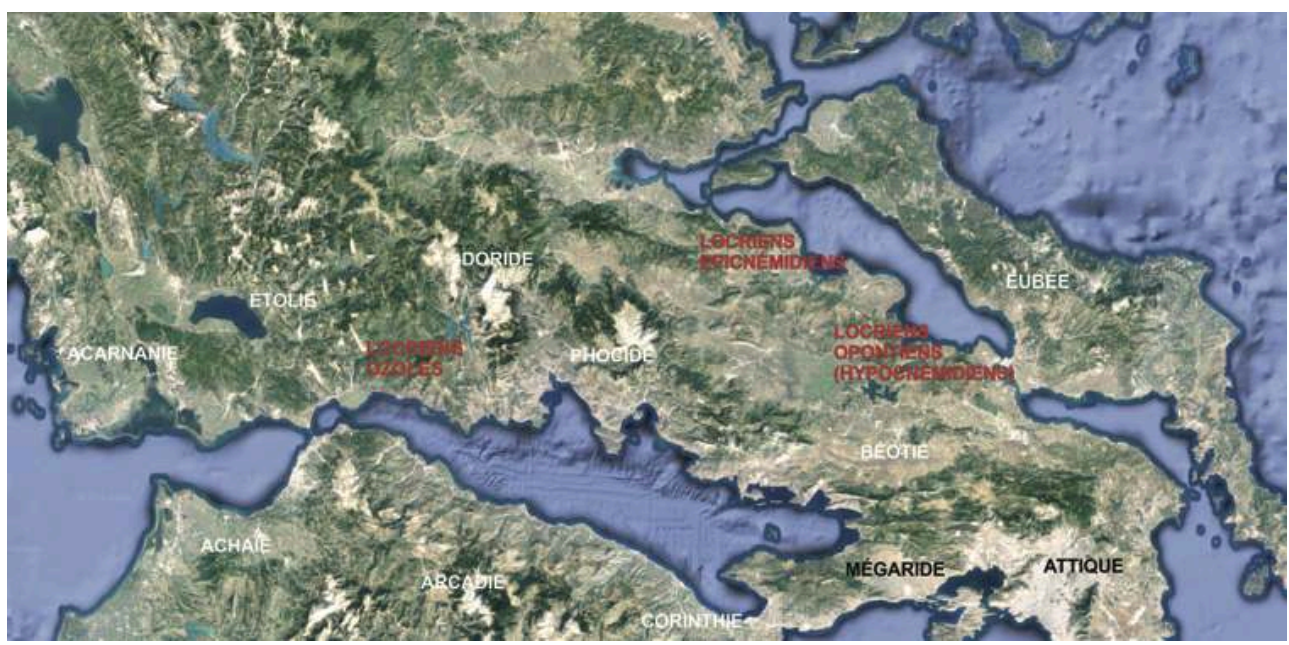

Image Google Earth, 2019

2 Le toponyme Locride peut définir trois régions différentes (fig. 1), liées entre elles par les récits mythiques de leur établissement, néanmoins complètement distinctes par leur fonctionnement administratif.

3 La Locride de l'Est occupe la zone côtière étroite sur le golfe Maliaque et le golfe Euboïque du Nord et est elle-même divisée en deux parties à l'est et à l'ouest du mont Cnémis, sur le point le plus étroit de la région, depuis l'époque où les Phocidiens se sont répandus vers le nord dans la région d'Élatée ${ }^{2}$. Ainsi les sources mentionnent les Locriens Opountiens ${ }^{3}$ ou Hypocnémidiens ${ }^{4}$ à l'est et les Locriens Épicnémidiens ${ }^{5}$ à l'ouest. La Locride de l'Est est entourée par la Béotie du côté sud-est tandis que le côté sud-ouest est limité par la Phocide et une partie de la Doride. Oponte fut la ville principale et éponyme de la Locride Opountia ${ }^{6}$. Au nord du mont Parnasse, la Locride Épicnémidienne occupe l'espace entre le mont Callidromos, le mont Cnémis et la mer 7 . Les coutumes funéraires de la région, connues et étudiées exclusivement par des fouilles de tombes antiques, attirent particulièrement notre attention. Les données recueillies ont permis de se faire une idée assez précise des rites qui sont en usage continu $\mathrm{du} \mathrm{VI}^{\mathrm{e}}$ au $\mathrm{II}^{\mathrm{e}}$ siècles av. J.-C. ${ }^{8}$ : les morts étaient déposés recroquevillés dans des pithoi funéraires la tête vers l'est et toutes les tombes étaient riches en offrandes.

4 À l'ouest du Parnasse, le paysage territorial n'est pas le même. La Locride de l'Ouest occupe le territoire ayant la Phocide à l'est, la Doride au nord et l'Étolie à l'ouest. À l'époque de sa plus grande étendue au IV siècle, la région occupe la zone côtière de Naupacte à l'ouest jusqu'au port de Kirrha et les limites de la terre sacrée d'Apollon à l'est. La Locride avait probablement occupé une partie du massif sud du Parnasse9. Cette région souffre des frontières peu ou mal définies au moins du côté nord et ouest et par conséquent la construction identitaire des cités qui la composent varie selon les époques et les sources qui en parlent, comme on verra par rapport à Naupacte.

5 Loin de la Grèce centrale, la cité des Locriens Épizéphyriens, appelée plus communément Locres, fut fondée sur la côte orientale de l'actuelle Calabre vers $700^{10}$. Elle fut gouvernée par une oligarchie appelée les Cents Maisons ${ }^{11}$. La Locride de l'Italie fut principalement connue pour la rigidité de ses lois ${ }^{12}$. Les deux traditions relatives à la 
fondation de Locres d'Italie sont attestées par le Ps.-Scymnos (v. 312-313 et 316-317), l'une l'attribuant aux Locriens Opontiens (Éphore F 138), l'autre aux Locriens Ozoles ${ }^{13}$.

6 Encore aujourd'hui dans la bibliographie le nom Locride se réfère à ces trois régions différentes décrites ci-dessus, mais aucune cohérence identitaire, cultuelle ou administrative n'est attendue. La construction de ce paysage territorial à divers endroits du monde antique à l'âge du fer est représentative d'un processus lent de différenciation des régions.

\section{Locriens Hespérioi ou Ozoles}

7 La Locride de l'Ouest (fig. 2) est l'une des rares régions à posséder une étude complète sur l'ensemble de son territoire, les vestiges antiques, les institutions et les événements historiques qui ont marqué le fil de son histoire. Dans les années 1930, Lucien Lerat entreprit des travaux de longue haleine sur l'ensemble de la région dans son étendue la plus vaste. Ses expéditions scientifiques ont été interrompues par la Seconde Guerre mondiale et finalement une monographie de deux volumes d'une ampleur remarquable a vu le jour en $1952^{14}$. À présent, cette publication reste la référence irremplaçable sur le sujet et ses travaux se complètent partiellement par les contributions de Denis Rousset $^{15}$ et surtout les fouilles de sauvetage menées par l'Éphorie des Antiquités. La région de la Locride, à l'ouest de Delphes, offre, par sa taille et ses monuments, l'occasion de traiter ces éléments l'un par rapport à l'autre et d'arriver ainsi à une image relativement complète de l'ensemble de la région et de son rôle de la Préhistoire jusqu'aux époques romaine et paléochrétienne. Pourtant tracer les étapes de la formation des centres urbains reste un défi. Dans notre démarche d'étudier l'organisation urbaine de la région, nous sommes appelés à interpréter les récits relatifs dans les traditions littéraires, prenant en compte le décalage chronologique et le contexte historique qui les sépare des « réalités » auxquelles ils se réfèrent.

Fig. 2. - La Locride de l'Ouest, cités principales et limites approximatives.

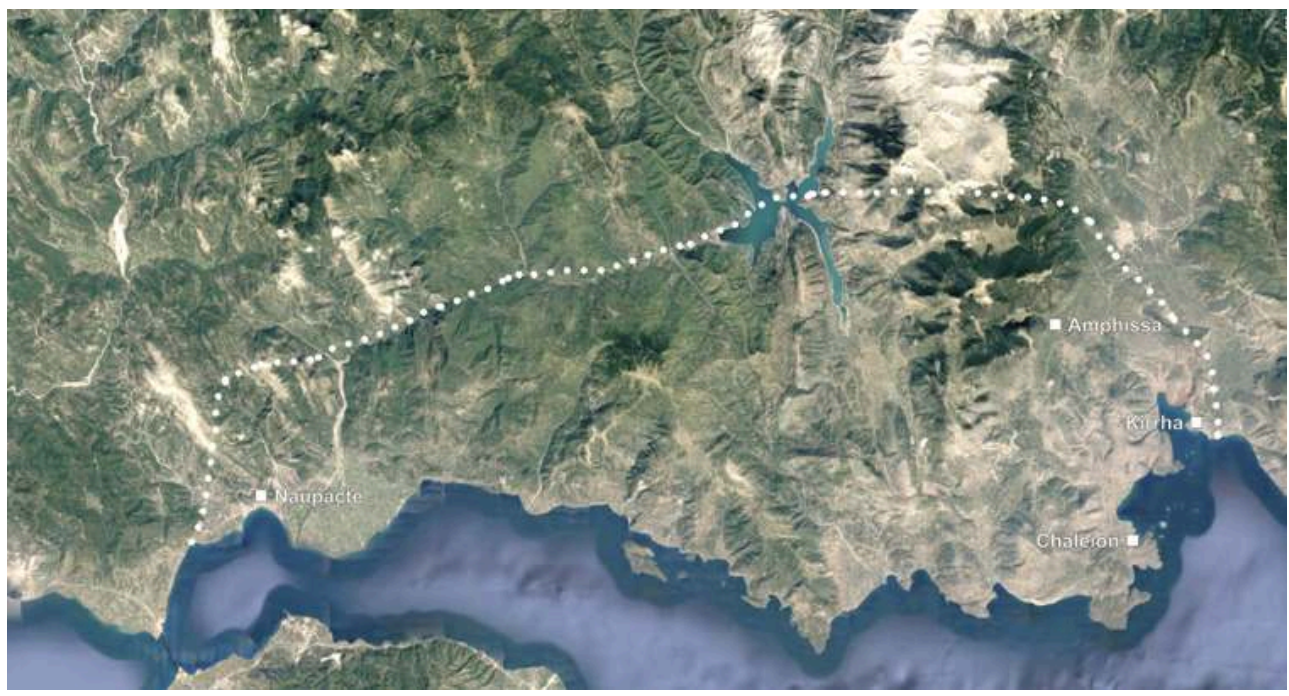

Image Google Earth, 2019.

Les Locriens de l'Ouest sont connus principalement sous le nom d'Ozoles $\left(\mathrm{O} \zeta{ }^{\prime} \lambda \alpha \mathrm{l}\right)^{16}$. Nombreuses sont les traditions qui expliquent cette appellation ${ }^{17}$, qui distingue les 
habitants de la Locride de l'Ouest des habitant de la Locride de l'Est. Les traditions sur

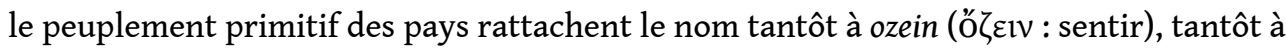
ozos (őłoc) qui signifie « rameau » ou «branche». Les versions concernant l'odeur des habitants ou des lieux se regroupent comme suit. Ozolai dériverait de l'odeur des femmes frappées de malédiction par Aphrodite ${ }^{18}$, les exhalaisons méphitiques des sources sulfureuses ou la senteur de certaines plantes ${ }^{19}$. D'après Strabon, dans le mont Taphiassos se trouvait le tombeau de Nessos et d'autres Centaures et l'odeur nauséabonde des eaux au pied de la montagne serait due à la décomposition des cadavres de ces monstres ${ }^{20}$. Nessos qui fut passeur ( $\left.\pi \rho \rho \theta \mu \varepsilon v ́ \varsigma\right)$ au fleuve Événos ${ }^{21}$, aurait été blessé par Héraclès et, d'après Pausanias, il ne mourut pas tout de suite, mais se réfugia dans la terre des Locriens où il pourrit sans être enterré (ö $\tau \alpha \varphi \circ \zeta)$ et transmit ainsi dans l'atmosphère une mauvaise odeur ${ }^{22}$. La puanteur des Locriens est également associée à l'odeur infecte des peaux de bêtes que les Locriens primitifs portaient à même le corps. Selon la tradition, les premiers hommes, autochtones, ne savaient pas tisser de vêtements et portaient des peaux de bêtes non traitées pour se protéger du froid, avec la partie poilue des peaux du côté extérieur pour être plus beaux ${ }^{23}$. Un autre passage décrit les émanations ou fumées et l'eau d'un fleuve local qui avaient une mauvaise odeur ${ }^{24}$. D'après d'autres sources, les Ozoles auraient au contraire reçu leur nom grâce à leur bonne odeur, leur pays étant rempli de fleurs. L'asphodèle poussait en abondance et quand il fleurissait il remplissait l'atmosphère de son parfum ${ }^{25}$. Le poète Archytas d'Amphissa explique le nom par la suave odeur des fleurs du pays : $\tau \grave{\alpha} v$

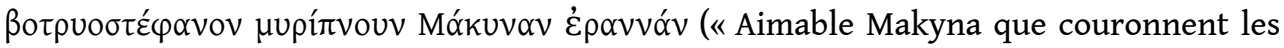
grappes et dont l'air est parfumé ») ${ }^{26}$.

Sans entrer dans le débat sur l'élaboration des mythes, il semble évident que ces explications insistent sur l'origine autochtone des Ozoles.

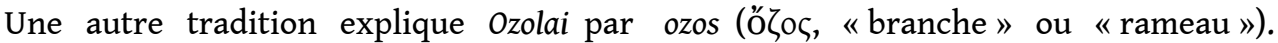
Pausanias raconte que la chienne d'Oresthée, fils de Deukalion et roi du pays, aurait donné naissance à un bout de bois au lieu d'un chien. Oresthée aurait planté le bois et on disait qu'au printemps une vigne avait poussé de celui-ci. Les hommes auraient pris leur nom des branches (öłouc) de cette plante ${ }^{27}$. Opous, le fils de Locros, eut un différend avec son père et après avoir attiré à lui une multitude de citoyens, consulta un oracle pour fonder une colonie. Le dieu dit de fonder une cité précisément là où il se

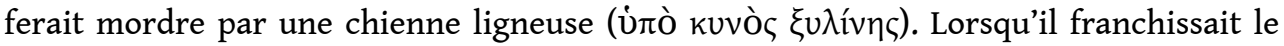
passage conduisant vers l'autre mer, il marcha sur une épine d'églantier. Gêné par la blessure, il resta plusieurs jours sur place, le temps qu'il lui fallut pour apprendre à connaître la contrée et fonder les cités de Physcos et d'Oiantheia, ainsi que le reste de celles où se sont établis les Locriens qu'on a qualifiés d'Ozoles ${ }^{28}$.

11 La tradition fait ainsi des Locriens de l'Ouest un rameau détaché des Locriens de l'Est et selon Lucien Lerat cette étymologie justifierait au mieux l'emplacement du peuple dans ce pays en donnant l'image d'une ethnie séparée en deux zones ${ }^{29}$. Les Locriens de l'Ouest sont dans certains cas nommés Hespérioi, par le nom de leur koinon, attesté à partir du IV siècle surtout par des sources épigraphiques ${ }^{30}$. Apparemment les Locriens de l'Ouest n'étaient pas connus d'Homère, qui cite seulement les Locriens qui habitaient en face de l'Eubée (Iliade, II, 535). Strabon (IX, 4, 7) veut expliquer qu'Homère dans ce vers fait une distinction implicite entre les Locriens de l'Est et les Locriens de l'Ouest. Plus précisément, l'arrière-pays de la région est difficilement accessible avant l'arrivée de Philippe II et le tracé de grands axes routiers. Les communications ont lieu 
principalement par voie maritime. Les centres urbains sont organisés le long de la côte à l'exception de la ville d'Amphissa et de Physkeis ${ }^{31}$ qui fut la capitale du koinon des Locriens Hespériens ${ }^{32}$. D'abord en 339, lors des guerres amphictyoniques ${ }^{33}$ et surtout au début $\mathrm{du} \mathrm{II}^{\mathrm{e}}$ siècle ${ }^{34}$, des conventions délimitent les frontières de manière bien plus nette $^{35}$. La Locride est organisée dans toute une série de bourgs, parmi lesquels les villes principales sont Amphissa, Chaleion et Naupacte.

\section{Naupacte}

La ville de Naupacte (fig. 3) se trouve sur la partie ouest du territoire et nous fournit des éléments intéressants pour la définition des centres urbains et de leurs territoires. Après la bataille de Chéronée, Philippe II, en avril-mai 338, s'en empara, massacra toute la garnison et remit la ville aux Étoliens qui la conservèrent jusqu'au jour où Auguste la rattacha à la colonie romaine de Patras ${ }^{36}$. Dans la littérature, Naupacte est souvent considérée comme étolienne ce qui crée des confusions ${ }^{37}$.

Fig. 3. - La ville de Naupacte actuelle avec l'emplacement des vestiges de l'âge du fer.

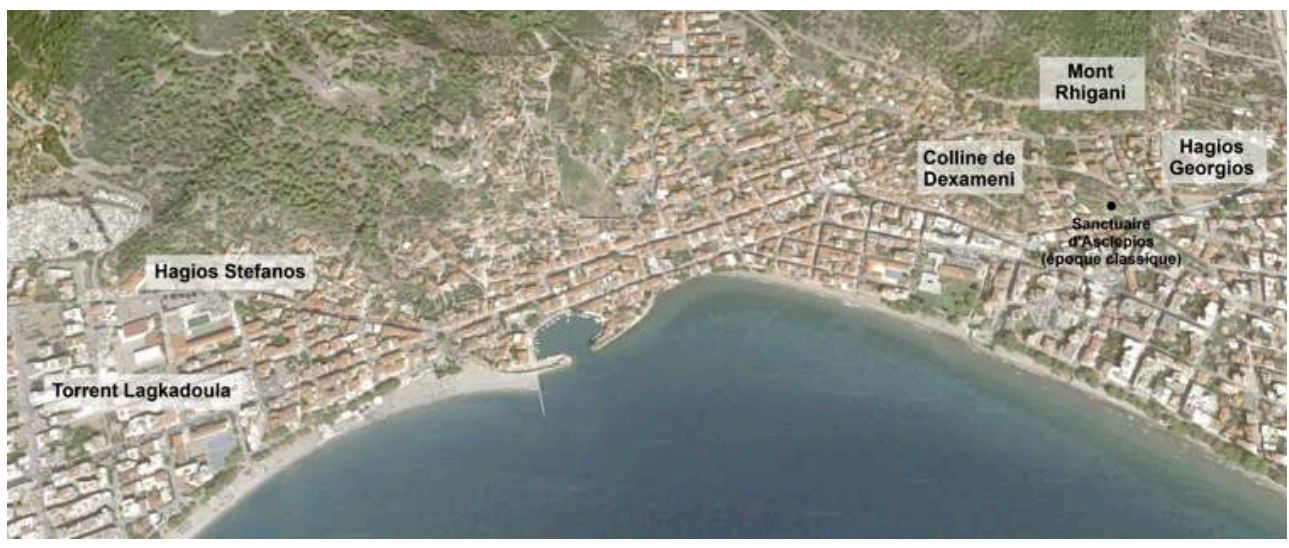

Image Google Earth, 2019.

13 Naupacte fut habitée quasiment sans rupture jusqu'à aujourd'hui et les vestiges ne sont pas facilement identifiables dans les couches perturbées. La ville connut une certaine prospérité à partir du XIII ${ }^{\mathrm{e}}$ siècle de notre ère sous la domination vénitienne et doit sa réputation à la bataille navale de Lépante en 1571 dans le contexte de la quatrième guerre vénéto-ottomane ${ }^{38}$. Dans les années 2000 lors de la grande effervescence urbanistique en Grèce, les tracés antiques et les vestiges des îlots d'habitat qui affleuraient parmi les constructions modernes ont été enfouis très rapidement, donc perdus pour la recherche et cela de manière particulièrement efficace. Les données qu'on peut néanmoins étudier sont connues grâce aux fouilles de sauvetage de l'Éphorie, mais il n'y a pas encore d'étude de l'ensemble du cadastre et les datations sont très vagues ${ }^{39}$.

\subsection{Vestiges archéologiques de l'âge du fer}

Les fouilles de sauvetage menées dans la ville de Naupacte et sa région environnante ont mis au jour des vestiges interprétés comme étant les noyaux d'agglomérations établies de manière éparpillée depuis l'époque mycénienne ${ }^{40}$. De l'âge du fer, nous 
avons pu distinguer trois sites principaux : le premier au nord-est et les deux autres aux limites nord-ouest de la ville hellénistique.

Sur les pentes ouest de la colline de Hagios Georgios (îlot 184 du cadastre) des vestiges ont été repérés sous les couches occupées par le cimetière hellénistique ${ }^{41}$. Les constructions s'étendaient apparemment sur une hauteur située dans le prolongement sud du mont Rhigani auprès de la colline actuelle Dexameni. Le sous-sol de la région est rocheux et avec une forte pente vers le sud. Les calcaires abondants sont visibles dans la partie nord, tandis que le reste du terrain est remblayé de terre rouge et rocailleuse. Des vestiges de bâtiments traversent le terrain du nord au sud, fouillé sur environ 8 mètres de long, constitués d'une série de blocs non taillés grands et moyens qui ont la forme d'analemma (mur de soutènement) puisqu'une partie se fondait sur le rocher en pente, tandis que le reste au sud se fondait sur les remblayages mentionnés. Ainsi, la différence de hauteur du sol diminue et aboutit à l'ouest du terrain à une surface plate dallée de petites pierres (de taille de roche). Seul le parement oriental de la surface extérieure est régulier. La construction continue vers le sud (îlot 183). Par endroits, on retrouve un sol pavé avec des pierres irrégulières. Les cavités naturelles du rocher cachaient une abondante céramique de bonne qualité et de l'outillage. Selon les fouilleurs, ces cavités rocheuses étaient probablement utilisées comme des silos. Aux îlots 186-187, les vestiges d'une partie de construction ovale ont été mis au jour, remployés dans des constructions du $\mathrm{v}^{\mathrm{e}}$ siècle av. J.-C.

Au sud-ouest de la fortification médiévale, dans la région de Hagios Stefanos ont été repérés, lors des fouilles pour la construction d'une école, quelques vestiges d'époque géométrique enfouis parmi des tombes hellénistiques et byzantines ${ }^{42}$. On peut reconstituer le plan de deux bâtiments construits sur des niveaux différents à cause du sol fortement escarpé. D'après la céramique abondante trouvée dans les remblais, le site date de la seconde moitié du viII ${ }^{\mathrm{e}}$ siècle. Le plan circulaire des bâtiments correspond probablement à des habitations ou à des entrepôts de stockage de céréales. Un peu plus loin, à 10-15 mètres du torrent Lagkadoula, des murs avec des pierres non taillées auraient servi à l'époque géométrique à l'aménagement des cours d'eau qui inondaient le site. Parmi les tombes du cimetière hellénistique, six pithoi funéraires datent de l'époque géométrique (première moitié $d u \mathrm{IX}^{\mathrm{e}}$-fin $\mathrm{VIII}^{\mathrm{e}}$ siècle), mais les éléments ne sont pas suffisants pour rétablir la forme du cimetière à cette époque.

Les deux noyaux que l'on qualifie d'urbanistiques repérés dans la ville de Naupacte datés de l'âge du fer, à environ deux kilomètres de distance l'un de l'autre, se trouvent à côté des sources d'eau et des zones de culture. Les constructions ne semblent pas avoir directement succédé à des aménagements plus anciens. Il est évident que la ville et son territoire sont habités sans régularité selon les besoins spécifiques de chaque époque sans obéir à un plan tracé sur la carte. L'édification de la ville et le plan urbain ne sont établis qu'à partir de la fin du IV siècle av. J.-C.

\subsection{Légendes et poésie de Naupacte}

Deux grandes légendes accompagnent l'histoire de la cité, celle des Héraclides et celle de la tombe du poète Hésiode.

19 Le récit mythique du « retour des Héraclides » désigne les expéditions des descendants du héros Héraclès : Eurysthée, le roi d'Argos, poursuivit non seulement Héraclès mais aussi ses enfants qui avaient été chassés de l'Argolide. Réfugiés en Attique dans la 
plaine de Marathon et après des affrontements avec les troupes d'Eurysthée et des tentatives infructueuses de retour dans leur pays d'origine, les Héraclides de la quatrième génération consultèrent de nouveau l'oracle. Ils se sont donc installés à Naupacte pour constituer une base maritime et de là ils entreprirent une expédition dans le Péloponnèse sous la direction du stratège $\mathrm{Oxylos}^{43}$. Naupactos doit alors son

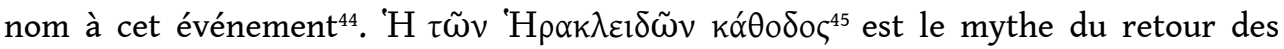
Héraclides dans le Péloponnèse et la récupération de leur héritage.

Le deuxième récit significatif concernant Naupacte est celui de la mort d'Hésiode. Le poète aurait été assassiné chemin faisant vers Naupacte, dans la ville locrienne auprès d'Oinoé ${ }^{46}$, par Ktimenos et Antiphos, fils de Ganyktor, parce qu'ils croyaient qu'Hésiode avait insulté leur sœur Kléméné. Les frères de la jeune fille le tuèrent et jetèrent son corps à la mer. Afin d'éviter la punition pour l'assassinat, ils s'enfuirent à Molykreion, mais comme ils ne respectèrent pas Poséidon, ils furent jugés et sévèrement punis ${ }^{47}$. Le corps du poète fut recueilli par un groupe de dauphins, qui le portèrent à l'entrée du golfe de Corinthe, à Rhion ou à Molycria. Trois jours après cet assassinat, les Locriens

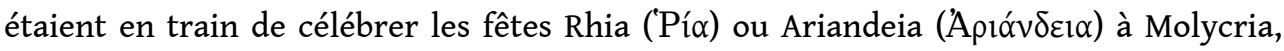
quand ils virent aborder le cadavre du poète avec son cortège marin. Ils se mirent aussitôt en quête des meurtriers, les découvrirent et les jetèrent à la mer. Ensuite, le corps d'Hésiode fut enseveli dans le sanctuaire proche de Zeus Némeios ou à Askra ; mais la place exacte du tombeau n'est pas connue: les habitants de Naupacte en gardèrent le secret, pour soustraire les cendres du poète aux Orchoméniens, qui voulaient les leur enlever. Par contre, les Orchoméniens étaient fiers de posséder la tombe d'Hésiode dans leur ville, soit pour avoir transporté les os d'Askra, soit pour les avoir volés à Naupacte ${ }^{48}$. Les os d'Hésiode auraient été transportés de Naupacte à Orchomène, sur l'ordre de la Pythie, afin de faire face à une maladie contagieuse ${ }^{49}$. Les Naupactiens conservaient alors la tombe vide. D'autres témoignages prétendent que les Orchoméniens auraient un jour atteint leur but, grâce à l'oracle de Delphes : sur le conseil de l'oracle, ils auraient suivi une corneille et, guidés par elle, trouvé le tombeau.

21 Cette histoire reflète de toute évidence la querelle ancienne entre les deux cités, Naupacte et Orchomène, qui se disputent l'honneur de posséder le tombeau d'Hésiode. Sans vouloir arbitrer sur ce conflit, nous pouvons du moins constater que la version dans laquelle Naupacte fonde ses prétentions contient de nombreux détails sur la légende: l'oracle mal compris, l'intervention miraculeuse des dauphins, l'arrivée du corps au milieu d'une fête, le tombeau caché, tous ces traits se retrouvent dans bien d'autres contes. D'autre part, nous pouvons aisément comprendre le point de départ de toutes ces inventions quand on s'aperçoit que le fils d'Hésiode et de la Locrienne séduite par lui porte le nom de Stésichore ${ }^{50}$. L'histoire des traditions relatives à Hésiode peut donc se reconstituer à peu près ainsi : à un certain moment, Naupacte eut un rôle de centre intellectuel, elle fut l'objet d'une épopée portant son nom, Naupaktia

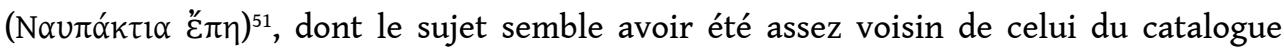
hésiodique. Il est dès lors naturel qu'elle ait cherché à récupérer Hésiode, comme également Stésichore. La légende était bien établie lorsque les Orchoméniens firent venir d'Askra les cendres d'Hésiode. Alors, naquirent d'autres versions de l'histoire, destinées à concilier les traditions contradictoires. Les unes devaient justifier les prétentions de Naupacte gardant jalousement un secret qu'Orchomène n'avait jamais pu lui arracher; les autres affirmaient au contraire que le dieu lui-même était intervenu en faveur des Orchoméniens pour leur promettre de ramener son corps de 
Locride. La tradition de la poésie d'Hésiode rend évidente l'influence qu'elle avait dans

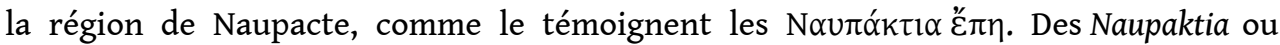
Naupaktika sont attribués à un certain Karkinos de Naupacte. Les poèmes ne portaient pas sur des sujets concernant Naupacte et sa région. Le titre témoigne plutôt du fait que ces poèmes étaient répandus dans la région de Naupacte ou étaient considérées en être originaires ${ }^{52}$. Pausanias les décrit comme parlant des femmes, une sorte de parallèle local aux Ehoiai d'Hésiode, avec une succession de généalogies ayant comme point de départ des héroïnes diverses. Ces poèmes contenaient au moins une narration héroïque, celle des Argonautes, et la version selon laquelle Jason aurait immigré vers Corcyre après la mort de Pélias.

\section{En guise de conclusion}

Afin de discerner les étapes de la formation des centres urbains, en prenant l'exemple de la Locride et de Naupacte, nous avons d'abord étudié la configuration géographique de la région, puis les légendes sur la cité. Malgré tous nos efforts, la définition de la région reste "flottante ». La ville de manière générale est une réalité urbanistique indissociable du territoire - de son territoire -, lui-même défini, au niveau géographique, par des frontières physiques et la revendication de certaines terres, et, au niveau ethnique, par des ancêtres communs ou par l'autochtonie des habitants. Même si les frontières se déplacent en raison de divers événements historiques, le territoire doit exister comme une unité, avec un caractère commun et un nom pour servir de cadre à l'organisation des terres et des agglomérations, ainsi que des populations qui commencent à se considérer comme issues des mêmes ancêtres ou comme autochtones.

Pour récapituler et conclure, la première ville de Naupacte, dont la forme urbaine exacte est inconnue, était bâtie au pied de la colline de Hagios Georgios. Il n'est pas nécessaire, au moins pour l'âge du fer, de chercher un plan urbain ou des structures architecturales élaborées, du moment que le territoire des cités - la région dont elles font partie - maintient une cohérence économique, politique, sociale et identitaire, une cohérence suffisante pour que les premières formes urbaines apparaissent. En d'autres termes, la ville et le territoire sont des réalités interdépendantes qui se structurent en même temps. Les récits qui évoquent la construction des navires des Héraclides pour la conquête du Péloponnèse et qui expliquent le nom de Naupacte supposent-ils une réalité topographique adaptée et un aménagement suffisamment organisé pour permettre une telle activité, ou bien faut-il se contenter d'exclure les mythes des sources qui rapportent la construction des cités et de leur territoire? Quel que soit le degré de fiabilité et d'exactitude, si on accepte l'hypothèse que la Locride de l'Ouest fut la colonie de la Locride de l'Est, on peut également imaginer comme objectif, à l'origine de cette expédition, l'occupation de la zone côtière le long du rivage vers l'ouest, où les eaux sont peu profondes et qui fera plus tard partie de la ville de Naupacte. La raison de cette colonisation n'est pourtant pas claire ${ }^{53}$. Il s'agissait probablement du besoin de renforcer un lieu stratégique, aux confins de l'Étolie, où la construction des navires est possible. 


\section{BIBLIOGRAPHIE}

\section{Abréviations}

- $A D=$ Archaiologikon Deltion.

- $\mathrm{BCH}=$ Bulletin de correspondance hellénique.

- $C I D=$ Corpus des inscriptions de Delphes, Paris, 1977-...

- EA = Ephéméris Archéologiki.

- FGrHist = JACOBY Felix, Die Fragmente der griechischen Historiker, Berlin, Weidmann, Leyde/ New York/ Cologne, E. J. Brill, 1923-1999.

- IG = Inscriptiones Graeciae, Berlin, G. de Gruyter, 1873-...

- P. Oxy = Papyrus Oxyrhynchus.

BECK Hans, « Ostlokris und die „Tausend Opuntier“. Neue Überlegungen zum Siedlergesetz für Naupaktos », ZPE, 124, 1999, p. 53-62.

BERNARD Nadine, « Aspects de la citoyenneté féminine en Grèce centrale à l'époque hellénistique », dans S. Crogiez-Pétrequin (éd.), Dieu(x) et Hommes. Histoire et iconographie des sociétés païennes et chrétiennes de l'Antiquité, Mont-Saint-Aignan, Publications de l'Université de Rouen et du Havre, 2005, p. 309-319.

BOULOGNE Jacques, Plutarque, Euvres morales, t. IV : [Traités 17-19], Conduites méritoires de femmes. Étiologies romaines. Étiologies grecques. Parallèles mineurs, Paris, Les Belles Lettres, 2002.

DUGAS Charles, « La mort du Centaure Nessos », REA, 45, 1943, p. 18-26.

FINGLASS Patrick, « Ethnic Identity in StesichorusAuthor(s) », ZPE, 182, 2012, p. 39-44.

FOSSEY M. John, The Ancient Topography of Opountian Lokris, Amsterdam, Gieben, 1990.

FREITAG Klaus, Der Golf von Korinth. Historisch-topographische Untersuchungen von der Archaik bis in das 1.Jh. v. Chr., Munich, Tuduv, 1999 (2000).

KOLONIA Rozina \& ROUSSET Denis, « Monuments funéraires de Locride occidentale », Chiron, 41, 2011, p. 181-216.

LEFÈVRE François, «L'Amphictionie de Delphes : mythe et réalité », Cahiers du centre Gustave Glotz, 6, 1995, p. 19-31.

LERAT Lucien, Les Locriens de l'Ouest, t. I : Topographie et ruines, t. II : Histoire, institutions, prosopographie, Paris, De Boccard, 1952.

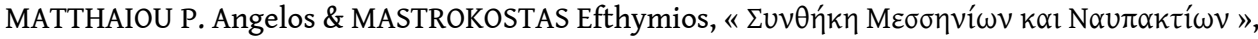
HOROS, 14-16, 2000-2003, p. 433-454.

MATTHEWS J. Victor, « Naupaktia and Argonautika », Phoenix, 31, 1977 (3), p. 189-207.

MAZON Paul (trad.), Hésiode, Théogonie ; Les travaux et les jours ; Le bouclier, texte établi et traduit par Paul Mazon, Paris, Les Belles Lettres, 1928.

MEIGGS Russell \& LEWIS David, A Selection of Greek Historical Inscriptions to the End of the Fifth Century BC, Oxford, Clarendon Press, 1988.

MILÁN Soledad, « Del Golfo de Corinto al cabo Cefirio: el viaje de los locrios y la fundación de Locris Epicefiria », Gerión, 31, 2013, p. 89-115.

MORIN Bernadette, «Du Tison au flocon : Méléagre et l'Héraclès des Trachiniennes ", L'Antiquité Classique, 76, 2007, p. 25-38. 
MULLIEZ Dominique, « Notes d'épigraphie delphique. I : Les affranchissements du théâtre et la chronologie delphique », BCH, 107, 1983, p. 429-450.

MULLIEZ Dominique, Recherches sur les actes d'affranchissement delphiques, thèse, Lille, Atelier national de reproduction des thèses, 1995.

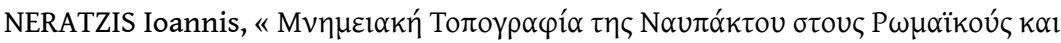

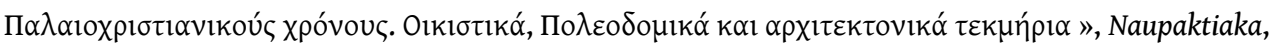
$\mathrm{I}_{2}, 2001$ (1998-1999), p. 43-118.

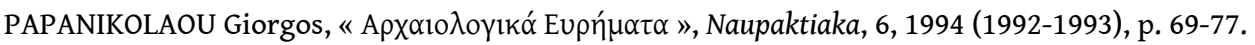

PARRA Maria Cecilia, « Locri », dans Bibliografia Topografica della colonizzazione greca in Italia e nelle Isole Tirreniche (BTCGI), 9, 1991, p. 191-249.

PASCUAL José \& PAPAKONSTANTINOU Maria-Foteini, Topography and History of Ancient Epicnemidian Locris, Leiden/Boston, Brill, coll. « Mnemosyne Supplements, History and Archaeology of Classical Antiquity, $362 », 2013$.

PAVESE Carlo Odo, «The Rhapsodic Epic Poems as Oral and Independent Poems », Harvard Studies in Classical Philology, 98, 1998, p. 63-90.

PEMBROKE Simon, « Locres et Tarente : le rôle des femmes dans la fondation de deux colonies grecques », Annales. Histoire, Sciences Sociales, 25, 1970 (5), p. 1240-1270.

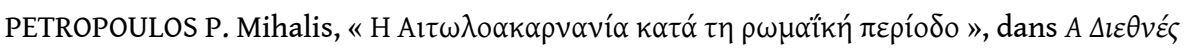

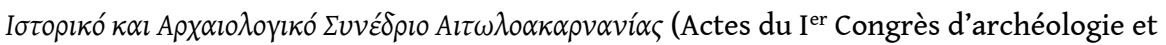
d'histoire d'Étoloacarnanie), Agrinio, 1991, p. 93-125.

PIGAILLEM Henri, La bataille de Lépante, 1571, Paris, Economica, 2017 (2éd.).

REDFIELD M. James, The Locrian Maidens, Love and Death in Greek Italy, Princeton, Oxford, 2003.

ROUSSET Denis, "Centre urbain, frontière et espace rural dans les cités de la Grèce centrale ", dans M. Brunet (éd.), Territoires des cités grecques, Paris, EFA-De Boccard, 1999, p. 35-77.

ROUSSET Denis, Le territoire de Delphes et la terre d'Apollon, Paris, École française d'Athènes, 2002. ROUSSET Denis, « West Lokris », dans M. H. Hansen \& T. H. Nielsen (éd.), An Inventory of Archaic and Classical poleis, Oxford, Oxford University Press, 2004, p. 391-398.

ROUSSET Denis, « Affranchissements de Physkeis en Locride occidentale », BCH, 130, 2006, p. 349-379.

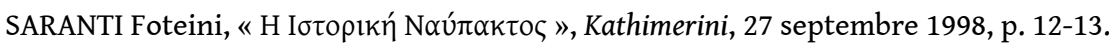

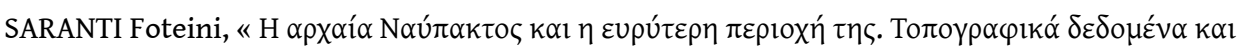

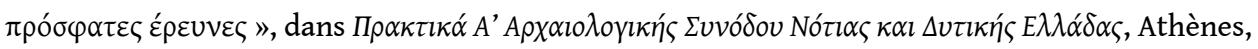
2006, p. 499-510.

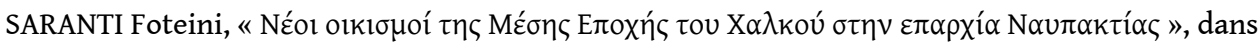
Mesoelladika : la Grèce continentale au Bronze Moyen, BCH, Suppl. 52, 2010, p. 259-267.

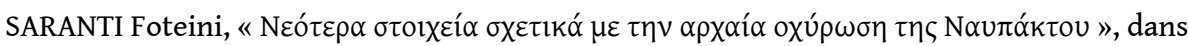

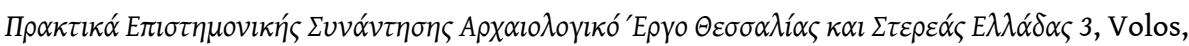

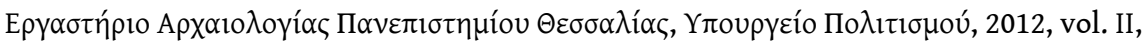
p. 763-771.

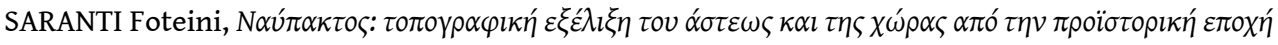

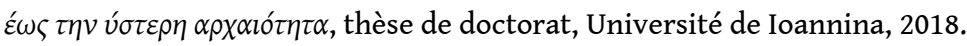




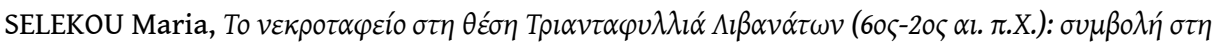

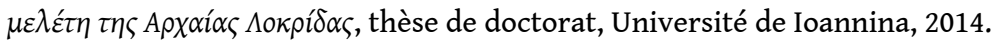

SELEKOU Maria, « Animal Terracottas from Children Graves at Opuntian Locris », dans A. Muller \& E. Lafli (éd.), Figurines de terre cuite en Méditerranée grecque et romaine, vol. 2 : Iconographie et contexte, Villeneuve-d'Ascq, Presses universitaires du Septentrion, 2015, p. 357-364.

SIMON Erika, « Oxylos und die dritte Generation der Herakliden », Numismatica e Antichità Classiche, 40, 2011, p. 161-177.

VAN COMPERNOLLE René, « La législation aristocratique de Locres Épizéphyrienne dite législation de Zaleukos », L'Antiquité Classique, 1981, 50, p. 759-769.

VAN EFFENTERRE Henri \& RUZÉ Françoise, Nomima : recueil d'inscriptions politiques et juridiques de l'archaïsme grec, t. I : Cités et institutions, Rome, EFR, 1994.

VANSCHOONWINKEL Jacques, « Des Héraclides du mythe aux Doriens de l'archéologie », Revue belge de philologie et d'histoire, 73, 1995 (1), p. 127-148.

VATIN Claude, « Le bronze Pappadakis, étude d'une loi coloniale », BCH, 87, 1963, p. 1-19.

WEST Martin, Greek Epic Fragments, Cambridge (Mass.), Harvard University Press, 2003.

WINIARCZYK Marek, « La mort et l'apothéose d'Héraclès », Wiener Studien, 113, 2000, p. 13-29.

WOHL Victoria, « A Tragic Case of Poisoning: Intention between Tragedy and the Law ", Transactions of the American Philological Association, 140, 2010 (1), p. 33-70.

\section{NOTES}

1. À l'exception de l'étude de Lucien Lerat (1952).

2. Vers la vallée de Dhipotamos et Longos. Cf. Strabon, IX, 3, 17.

3. Strabon, IX, 4,1 ; IX, 2, 42.

4. Hérodote, VI, 703, 8 ; Xénophon, Helléniques, IV, 2, 17 ; Pausanias, I, $23,4$.

5. Strabon, IX, 3, 1; Pausanias, X, 13, 4 ; Apollodore, II, 154 ; Pline l'Ancien, IV, 7, 27.

6. Fossey (1990) consacre une monographie à cette région dans le but de rendre une image complète de celle-ci comme Lerat (1952) l'a fait pour les Ozoles. La démarche scientifique est pourtant différente et l'intérêt porte davantage sur la géographie et la répartition des vestiges sur la carte que sur la prosopographie et l'histoire institutionnelle du pays. Avec ses études sur la Phocide de l'Est et la Béotie, Fossey complète l'image archéologique de la Grèce centrale.

7. Pour une étude récente sur l'ensemble de la région avec bibliographie, voir Pascual \& Papakonstantinou (2013).

8. Toutes les données sur le sujet proviennent de fouilles de sauvetage de l'Éphorie. Selekou (2014 et 2015) en fait une excellente synthèse.

9. Strabon, IX, 3, 1 (C 416-418).

10. Strabon, VI, 1, 10 ; Pline, III, 95. D'après Thucydide, VI, 4, 4 et Pausanias, III, 3, 1, la fondation de Locres date de la première guerre de Messénie en 734/733. Mais d'après la Chronique d'Eusèbe la cité des Locres serait fondée en 679/678 ou 673/672. Voir récemment Milán (2013).

11. Polybe, Histoires, XII, 5, 6. Les «Cent Maisons » sont les descendants de la noblesse locrienne de lignée maternelle. Les Locriens avaient participé aux côtés des Spartiates à la guerre contre les Messéniens et l'absence des hommes se prolongeant, un certain nombre de femmes s'unirent à leurs esclaves. Lors du retour des soldats, la situation troublée s'était terminée par le départ des esclaves, des femmes adultères et des bâtards, qui étaient venus s'établir à Locres. Cette fondation coloniale rappelle celle de Tarente et les Partheniai que les femmes spartiates avaient 
conçus également de leurs esclaves. Les deux cas d'installation coloniale à Locres et à Tarente ont donné lieu à une abondante bibliographie sur le matriarcat en Grèce ancienne : Bernard (2005) ; Pembroke (1970) ; Lerat (1952, II, 137-139).

12. Démosthène, Contre Timocrate, 139-143. Voir Van Compernolle (1981).

13. Le Ps.-Scymnos prend parti pour Éphore ; Strabon pour l'autre tradition (VI, 2, 7, C 259), cf. Parra (1991).

14. Lerat (1952, I et II). En 2008 et 2009, la traduction en grec de ses travaux a vu le jour avec un commentaire et une mise à jour bibliographique et archéologique.

15. Voir par exemple Rousset (2002) sur le territoire de Delphes et les confins avec la Locride; Rousset (1999) fournit une étude de localisation des frontières des cités et de leurs territoires s'appuyant sur les enceintes fortifiées ; Rousset (2004) répertorie toutes les poleis de la Locride de l'Ouest attestées par l'archéologie et la littérature; Rousset (2006) publie une étude d'affranchissement à Physkeis. Kolonia et Rousset (2011) publient une étude sur les monuments funéraires de la région avec beaucoup de réflexions topographiques et la bibliographie antérieure. Voir aussi les travaux de Mulliez (1995 et 1983) sur les actes d'affranchissement delphiques, où la présence des Locriens est également étudiée.

16. Les Ozoles sont mentionnés chez Hérodote, VIII, 32 ; Thucydide, I, 5, 3 ; Xénophon, Helléniques, IV, 2,17 ; Ps.-Scylax, 36.

17. Pausanias $(\mathrm{X}, 38,1-3)$ regroupe les traditions principales.

18. Servius, ad Virgile, Énéide, III, 399.

19. Pausanias, $X, 38,2$.

20. Strabon, IX, 4, 8 (C 427); Myrsilos de Méthymne (III ${ }^{\mathrm{e}}$ siècle av. J.-C.) cité par Antigonos de Carystos: FGrHist III B, 477, F 6 (7), qui atteste la même tradition pour expliquer le nom des Locriens Ozoles. Pour Apollodore, II, V, 4, Nessos ne fut pas le seul centaure à avoir échappé. Cf. Plutarque, Questions grecques, 15 ; Servius, ad Virgile, Énéide, III, 399.

21. Événos est un torrent qui porte aujourd'hui le nom de Phidaris et se jette dans le golfe de Patras. Cf. Pausanias, X, 38, 2.

22. La mort du centaure Nessos est connue dans plusieurs versions dont la plus célèbre est celle de Sophocle dans Les Trachiniennes, 555-577 : Héraclès, voyageant en compagnie de Déjanire, est arrêté par le fleuve Événos. Le centaure Nessos, qui fait franchir le fleuve aux voyageurs en les portant dans ses bras en échange d'un salaire, offre d'aider Déjanire à traverser ; mais, parvenu au milieu du fleuve, il tente de la violer. Héraclès le tue à l'aide d'une flèche enduite du poison de l'Hydre de Lerne. Nessos agonisant conseille à Déjanire de recueillir son sang et de l'offrir plus tard à Héraclès en guise de philtre d'amour, pour s'assurer à jamais de sa fidélité. Déjanire obéit et enduit sa tunique du sang du centaure. La tunique a pris le poison de l'hydre et, lorsqu'Héraclès s'en revêt, il meurt dans d'horribles souffrances. Voir aussi Dion Chrysostome, LX, Nessos ou Déjanire; Dugas (1943) ; Miniarczyk (2000, avec bibliographie). Sur le remaniement du mythe d'un point de vue juridique ou théâtral, voir Morin (2007) et Wohl (2010). Plus récemment, Finglass (2012) étudie le fragment d'un poème de Stésichore (P. Oxy. 2359 fr. 1, col. ii) relatif au mythe en question, analyse son élaboration à l'époque archaïque et explique la référence aux peuples concernés, surtout les Locriens.

23. Pausanias, X, 38, 3 ; Plutarque, Questions grecques, 15 (C 294F).

24. Pausanias, $X, 38,2$.

25. Ibid.

26. Trad. Boulogne (2002). Ce vers en hexamètre est cité par Plutarque, Questions grecques, 15

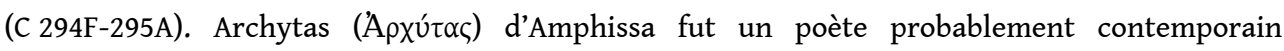
d'Euphorion de Chalcis (environ 300 av.J.-C.) d'après le passage d'Athénée (Deipnosophistai,

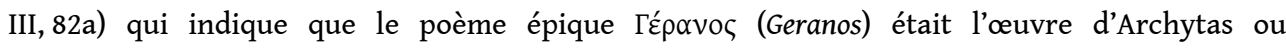
d'Euphorion. Makyna était située à l'est du mont Taphiassos.

27. Pausanias, $\mathrm{X}, 38,1$.

Gaia, $22-23$ | 2020 
28. Plutarque, Questions grecques, 15 (C 294E).

29. Lerat (1952, II, 5) : «les Locriens Ozolai sont des colons des Locriens de l'Est». Cette interprétation est également renforcée par l'inscription sur deux lamelles en bronze (IG IX, $1^{2}, 3$, $718,1.1)$, où Naupacte est citée comme destination de colonie des Locriens Hypocnémidiens : غ่v

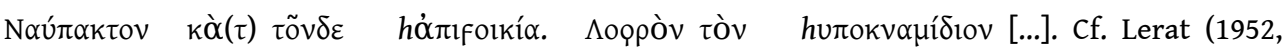
II, 29-31) ; Matthaiou-Mastrokostas (2000-2003, 447-448); Beck (1999). La loi coloniale sur le partage de certains territoires (IG IX, $1^{2}, 3,609$ ), inscription sur bronze publiée par Pappadakis (EA 1924 [1926], 119-141) - considérée comme non locrienne par Lerat (1952, II, 9-10), qui l'attribue sous réserve à l'Étolie - est pourtant réintégrée dans la Locride par Freitag $(2000,69$ et note 344) ; Meiggs \& Lewis (1988, n 13) ; Vatin (1963) ; Effenterre \& Ruzé (1994, nº 44) ; Hansen (1987, 89-90).

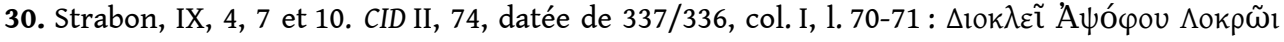

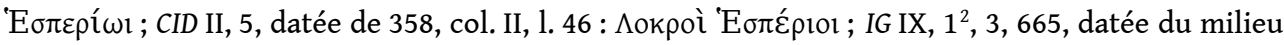

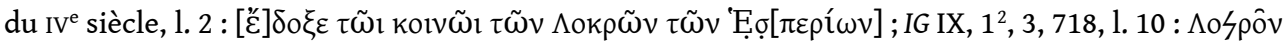

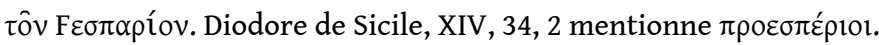

31. Plutarque, Questions grecques, 15 (C 294E), écrit que Locros fut le fils de Physcios et le père d'Opous. L'inscription IG IX, $1^{2} 3,665$ datée du milieu du IV siècle et trois inscriptions du milieu

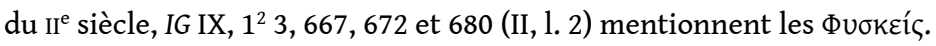

32. Lerat (1952, I, 123-137 ; II, 97-98).

33. «Quatrième guerre sacrée » en 339-338, cf. Lefèvre $(1995,27)$.

34. Freitag (2000, 87 et 92).

35. Le territoire est doté d'une série de tours qui ne font pas forcement partie de la fortification des agglomérations. Saranti (2012) pour Naupacte fournit une mise à jour archéologique de l'état des vestiges. Il nous est difficile de dater et de reconstituer le système dont elles faisaient probablement partie, mais il semble cohérent qu'elles contribuent à la structuration de l'ensemble de la région.

36. Théopompe, FGrHist $115 \mathrm{~F} 235$.

37. Par exemple Ps.-Scymnos (478-479) se réfère à la ville de Naupacte, fondée par Téménos, à l'est de Rhion, comme faisant partie du territoire de l'Étolie et les Locriens Ozoles seraient des colons de la Locride de l'Est. Cf. Petropoulos (1991); Freitag $(2000,89)$.

38. Certains historiens estiment qu'il s'agit de la bataille navale la plus importante de l'histoire par ses conséquences. Parmi une bibliographie très riche sur le sujet nous citons la publication récente de Pigaillem (2017). Le déroulement des événements est connu principalement par un texte contemporain anonyme, intitulé La bataille de Lépante, traduite de l'espagnol par Jean Pagès (2011).

39. Saranti (2018) publie sa thèse de doctorat sur la ville de Naupacte et entreprend la synthèse des vestiges des fouilles de sauvetage menées par l'Éphorie, qui ne sont pas encore publiées et restent partiellement étudiées, avec des données datées de manière approximative et pas encore systématiquement répertoriées. Voir aussi Neratzis (2001), Papanikolaou (1994) et Saranti (1998 et 2010) pour les vestiges de l'époque mycénienne dans la région environnante.

40. Saranti $(2018,127-177)$ dresse plus précisément l'état des vestiges actuellement connus pour l'âge de fer.

41. L'occupation du site peut être attestée depuis le $\mathrm{XV}^{\mathrm{e}}$ siècle av. J.-C. non sans certaines discontinuités. Cf. Saranti $(2018,106)$; Alexopoulou (AD 49, 1994, Chron., 243-244) ; BCH (1999, Chron., 725).

42. Saranti $(2018,136-140)$. Les fouilles du site ne sont pas encore publiées. Les parements ont donné la fausse impression aux fouilleurs qu'il s'agissait des périboles ou des murs de soutènement. 
43. Vanschoonwinkel (1995) offre une synthèse des récits du mythe et des interprétations modernes dans la bibliographie. Simon (2011) revient sur l'expédition d'oxylos à propos d'un cratère en cloche attique (420-410) représentant ce mythe de fondation pour certaines installations dans le Péloponnèse.

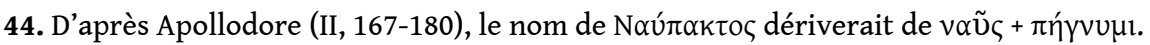

45. L'expression fait son apparition au IV siècle chez Éphore (FGrHist 70 F18) et est reprise notamment chez Diodore de Sicile (IV, 58, 4), Apollodore (II, 8, 2) et Pausanias (IV, 3, 3 ; V, 3, 5 ; VIII, 5,1). Si la constitution de cette légende expliquant certaines installations dans le Péloponnèse date $\mathrm{du} \mathrm{IV}^{\mathrm{e}}$ siècle, elle coïncide avec l'époque de l'édification et de l'organisation urbanistique de la ville de Naupacte.

46. Aujourd'hui Magoula, un peu au nord d'Eupalion. Thucydide (III, 96) indique que le poète Hésiode est mort dans le sanctuaire de Zeus de Némée.

47. Pausanias, IX, 31, 6 ; Suidas, IV, 764, 1. 11.

48. Plutarque, Banquet des sept sages, 19, 162e-f. Cf. Anthologie Palatine, VII, 55 (v. 1-3).

49. Pausanias, IX, 38, 3-4; Dispute d'Homère et Hésiode, 1.350-357. Pour l'épigramme citée par Pausanias, voir aussi Anthologie Palatine, VII, 54. A $\gamma \omega ́$ v (Dispute d'Homère et Hésiode) est le récit du tournoi poétique entre Homère et Hésiode qui contient aussi des renseignements sur la mort d'Hésiode. Ce dernier gagna et dédia un trépied à Delphes. La Pythie l'avertit d'éviter le « Néméion ». Hésiode s'était donc écarté avec soin de la route du Péloponnèse, puisqu'il croyait qu'il s'agissait de la ville de Némée (Dispute d'Homère et Hésiode, 1. 221-222).

50. Tzetzès, p. 50, 1. 25. Voir Mazon (1928, VII-XIV). Voir aussi ci-dessus note 22 et Finglass (2012) sur le fragment de Stésichore.

51. Pausanias, $X, 38,10-11$. Charon de Lampsacos, auteur de la fin du ve siècle av. J.-C., attribuait les Naupaktia à Karkinos de Naupacte, tandis que d'autres les attribuaient à un auteur de Milet. Voir West $(2003,33)$. La plupart des fragments proviennent des scholies d'Apollonios de Rhodes et quelques vers conservés sont relatifs au voyage des Argonautes; il était œuvre de généalogie.

52. Comme c'est le cas des Chants cypriens.

53. Voir ci-dessus note 29.

\section{RÉSUMÉS}

La Locride de l'Ouest et la ville de Naupacte sont des case studies révélateurs des problèmes auxquels l'historien se heurte pour cerner et décrire le moment de l'émergence des centres urbains, ainsi que la structuration de leurs régions. Le nom de Locride peut définir trois régions différentes, liées entre elles par les récits mythiques de leur naissance et complètement distinctes par leur fonctionnement administratif. La Locride de l'Ouest, entre la Phocide et l'Étolie, est occupée par une série de kômai, parmi lesquelles Naupacte se trouve à l'extrémité ouest de la région. Malgré la pénurie de vestiges archéologiques qui auraient pu nous offrir une reconstitution complète de sa forme urbaine à l'âge du fer, nous pouvons entrevoir les étapes de la formation des sites en prenant en considération les sources littéraires et l'organisation de l'ensemble du territoire.

West Locris and Nafpaktos are relevant case studies for the emergence of urban and territorial structures in the Iron Age. The name Locris corresponds to three different regions, with common myths for their formation and completely distinct by their administrative functioning. 
West Locris, covering the territory between Phocis and Aetolia, is composed of a series of komai, among which Nafpaktos that occupies the western edge of the region. Despite the shortage of archaeological remains that could have offered us a complete reconstruction of its urban form in the Iron Age, we propose a glimpse of the progressive urbanization, taking into account the literary sources and the organization of the whole territory in terms of land use and cultural or political exchange.

INDEX

Mots-clés : Locride, Ozoles, Naupacte, Naupaktia

Keywords : West Locris, Ozolai, Nafpaktos, Naupaktia

\section{AUTEUR}

\section{DESPINA CHATZIVASILIOU}

Collège de France

despina.chatzivasiliou@college-de-france.fr 